Ann. Biol. anim. Bioch. Biophys., I976, 16 (6), 79ז-794.

\title{
RÔLE JOUÉ PAR LA SURRÉNALE DANS LE DÉCLENCHEMENT DE L'OVULATION PAR L'HÉMICASTRATION CHEZ LA RATTE
}

\author{
Jacqueline ROOS, M. ROOS et Cl. ARON \\ avec la collaboration technique de Christine Lazarus et de Viviane Siefrer \\ Institut d'Histologie et ERA 566 du C. N. R. S., \\ Faculté de médecine, \\ 4, rue Kirschleger, \\ 67085 Strasbourg Cedex

\begin{abstract}
RÉSUMÉ
L'hémicastration, pratiquée le matin du diœstrus III, peut avancer l'ovulation de 24 heures, chez des rattes à cycles de 5 jours. Une simple traction faite sur le méso-ovaire exerce également cet effet qui apparaît supprimé par la surrénalectomie. Des laparotomies sans manipulation de l'ovaire ou de simples contentions sous éther, effectuées en dioestrus III, ne provoquent pas de modification du rythme cestral, alors que, réalisées en procestrus, elles raccourcissent le cycle cestral de 24 heures, en un pourcentage notable de cas.
\end{abstract}

\section{INTRODUCTION}

L'hémicastration entraîne une ovulation anticipée quand elle est pratiquée en diostrus III de cycles de 5 jours chez la Ratte (Roos et Roos, I966, $a$ et $b$ ). La surrénale peut être impliquée en procstrus dans le déclenchement de l'ovulation par le stress chirurgical (LAWTON, I972). Nous avons donc recherché si la surrénalectomie pouvait prévenir l'effet de l'hémicastration, d'autres travaux ayant montré qu'elle était à même d'intervenir sur le moment de l'ovulation (FEDER et al., I97I) et donc, sur la durée du cycle œstral (BUFFLER, I973; Roos, non publié).

\section{TECHNIQUES EXPÉRIMENTALES ETT MATÉRIEI UTILISÉ}

Nous avons utilisé en tout 142 rattes Wistar de la souche WI, âgées de 3 à 4 mois, pesant I 90 à $210 \mathrm{~g}$ et présentant toutes des cycles de 5 jours. Soumises à l'éclairement naturel, elles recevaient un aliment composé et de l'eau ad libitum. L'eau de boisson des femelles surrénalectomisées était salée à 7 p. I ooo. 
Les femelles ont été divisées en 2 groupes.

Le premier groupe a comporté 3 lots de 16 rattes chacun. Les femelles du rer lot ont été hémicastrées en dicestrus III, entre 10 et I I heures; celles du $2^{\mathbf{e}}$ lot ont été soumises, au même stade que celles du lot précédent à une hémicastration simulée, consistant en une traction exercée sur le méso-ovaire sans ablation de l'organe; celles du $3^{\text {e }}$ lot, qui avaient été surrénalectomisées 2 cycles auparavant en procestrus, ont été hémicastrées dans les mêmes conditions chronologiques que celles du ier lot.

Le second groupe a été divisé en 4 lots de rattes auxquelles nous avons fait subir tout d'abord, en dicestrus III ou en prooestrus, une opération simulée. Fn diøestrus III, 20 et I9 femelles respectivement ont été soumises sous anesthésie à l'éther, à une simple contention pendant I/4 d'heure (temps correspondant à une surrénalectomie) ou à une surrénalectomie à blanc, réduite à une incision bilatérale des plans cutanés et musculaires. En procstrus, 29 et 26 femelles respectivement ont subi le même traitement. Les rattes ayant maintenu, dans ces conditions, leur rythmicité de 5 jours ont été hémicastrées, 3 à 4 cycles après l'intervention, en dicestrus III, entre Io et I I heures, comme celles du I er groupe.

Toutes les femelles ont été sacrifiées en proøstrus et leurs ovaires prélevés aux fins d'un examen histologique.

\section{RÉSULTATS}

Le tableau I montre que l'ovulation ou la lutéinisation suscitée par l'hémicastration (I3/I6) ne se manifeste plus chez les rattes surrénalectomisées (2/I6) et que la simulation de l'hémicastration, chez des rattes ayant conservé leurs glandes surrénales, est à même de déclencher l'ovulation (8/16).

Le tableau 2 montre tout d'abord que les simulations opératoires effectuées en procstrus ont entraîné plus fréquemment un raccourcissement du cycle que celles réalisées en diœstrus III. L'analyse statistique d'un plan factoriel, rendu possible par l'égalisation des effectifs à Ig et transformation angulaire des pourcentages, confirme ce résultat. Il apparaît ensuite que l'hémicastration a accru, de façon statistiquement significative (même modèle d'analyse que ci-dessus), l'ovulation ou la lutéinisation chez les rattes ayant maintenu un rythme de 5 jours après manipulation en diœstrus III, par rapport à celles ayant conservé ce rythme après manipulation en proœstrus.

\section{DISCUSSION DES RÉSULTATS}

Nos résultats indiquent clairement que l'ovulation et la lutéinisation déclenchées par l'hémicastration, au cours de cycles de 5 jours chez la Ratte, mettent en jeu les glandes surrénales.

Ils montrent également que les effets de l'ablation de l'ovaire ne sont pas la conséquence d'un déséquilibre hormonal lié à l'hémiovariectomie étant donné qu'une simple traction exercée sur le méso-ovaire a suffi à déclencher l'ovulation ou la lutéinisation dans les conditions chronologiques où l'hémicastration induisait cet effet. $L_{\text {a }}$ question se pose donc de savoir si l'hémicastration ne suscite pas un stress neurogène prenant son origine au niveau même de l'ovaire. Ces observations confirment celles effectuées antérieurement chez le Cobaye par ARON et al. (I948). Cette hypothèse apparaît d'autant plus plausible qu'en l'absence de traction exercée sur l'ovaire les opérations simulées, ou les contentions sous éther, effectuées en diostrus III, n'ont provoqué aucun raccourcissement du cycle témoignant de l'avancement de l'ovulation. 
TABLEAU I

Effets ovulatoires de l'hémicastration, effectuée en dioestrus III, de cycles de 5 jours, chez des rattes intactes ou préalablement surrénalectomisées en prooestrus

\begin{tabular}{c|c}
\hline Traitement & Fréquences ovulatoires \\
\hline Hémicastration vraie & $13 / 16$ \\
& $(81 \%)$ \\
\hline Hémicastration simulée & $8 / 16$ \\
& $(50 \%)$ \\
\hline Hémicastration vraie & $2 / 16^{*}$ \\
après surrénalectomie & $(12 \%)$ \\
\hline
\end{tabular}

* La inéthode de Newman-Keuls, après transformation angulaire des pourcentages, montre que cette fréquence diffère significativement des deux autres $(p<0,05)$.

\section{TABLEAU 2}

Effets du stress, à différents stades de cycles de 5 jours, sur la durée du cycle oestral et effets ovulatoires de l'hémicastration, en dioestrus $I I I$, chez des rattes ayant maintenu leur rythme initial de 5 jours

\begin{tabular}{c|c|c}
\hline \hline Traitement & $\begin{array}{c}\text { Fréquences } \\
\text { de raccourcissement } \\
\text { du cycle de 24 heures } \\
\text { a la suite du stress* }\end{array}$ & $\begin{array}{c}\text { Fréquences } \\
\text { ovulatoires après } \\
\text { hémicastration** }\end{array}$ \\
\hline $\begin{array}{c}\text { Contentions sous éther } \\
\text { en dioestrus III }\end{array}$ & $\begin{array}{c}2 / 20^{a} \\
(10 \%)\end{array}$ & $\begin{array}{c}11 / 16^{e} \\
(68 \%)\end{array}$ \\
\hline $\begin{array}{c}\text { Surrénalectomie } \\
\text { simulée en dicestrus III }\end{array}$ & $\begin{array}{r}2 / 19^{b} \\
(11 \%)\end{array}$ & $\begin{array}{c}8 / 16^{f} \\
(50 \%)\end{array}$ \\
\hline $\begin{array}{c}\text { Contention sous éther } \\
\text { en procestrus }\end{array}$ & $\begin{array}{r}7 / 16^{g} \\
(45 \%)\end{array}$ \\
\hline $\begin{array}{c}\text { Surrénalectomie } \\
\text { simulée en pronestrus }\end{array}$ & $\begin{array}{c}13 / 29^{c} \\
(38 \%)\end{array}$ \\
\hline
\end{tabular}

$* a, b$ contre $c, d: \mathrm{F}_{\infty}^{1}=10,70 ; p<0,01-a, c$ contre $b, d: \mathrm{F}_{\infty}^{1}=$ 0,$22 ; N S$.

Interaction : $\mathrm{F}_{\infty}^{1}=0,22 ; \mathrm{NS}$.

** $e, f$ contre $g, h: \mathbf{F}_{\infty}^{1}=4,27 ; p<0,05-e, g$ contre $f, h: \mathbf{F}_{\infty}^{1}=$ 2,45 ; NS.

Interaction : $\mathrm{F}_{\infty}^{1}=0,01 ; \mathrm{NS}$. 
Ainsi à ce stade du cycle au moins, l'éther, contrairement à ce qui a été établi par AJIKA et al. (I972), mais conformément aux observations de LAWTON (I972), n'est pas capable en soi de provoquer une décharge suffisante de $L H$ pour déclencher prématurément la ponte ovulaire ou la lutéinisation. Il n'en va pas de même en proœstrus où une opération simulée, sans manipulation ovarienne, se montre à même de provoquer un raccourcissement du cycle en 42 p. Ioo des cas.

Tout se passe donc comme si la Ratte était plus sensible en proœstrus qu'en dicstrus III de cycles de 5 jours à un stress comportant, soit une simple contention sous éther, soit une opération à blanc. Ce résultat, en accord avec ceux de LAwToN (I972), explique que des femelles, ayant subi les manipulations décrites ci-dessus en diœstrus III, aient réagi plus fréquemment à une hémicastration ultérieure que des rattes ayant subi ces manipulations en proøstrus, où, en effet, les plus sensibles d'entre elles au stress se sont éliminées de l'expérience par un raccourcissement de leur cycle à la suite de la première manipulation

Reçu pour publication en mars 1976.

\section{SUMMARY \\ ROLE OF THE ADRENAL GLAND IN TRIGGERING OVULATION BY HEMICASTRATION IN RAT}

It has been observed that hemiovariectomy practised the morning of diestrus III of 5 -day cycles triggered ovulation in rat (Roos and Roos, 1966). This report shows that the adrenals play a role in $13 / 16$ cases where the females are hemiovariectomized and in only $2 / 16$ cases where they are surrenalectomized then hemiovariectomized. Effects of hemiovariectomy are related to manipulation of the ovary because in 8/16 cases females ovulate or luteinize early when simple traction is applied on the meso-ovary. The role of ovary manipulation is also confirmed by laparotomy without meso-ovary traction or by simple retention under ether; both fail to advance ovulation in diestrus III. In $42 \mathrm{p}$. Ioo of proestrous rats undergoing these two kinds of sham operations stress threshold of the shortened cycle varies from day to day. Among females maintaining the 5-day rhythm after these sham operations, eventual hemiovariectomy triggers ovulation in $19 / 32$ rats manipulated in diestrus III and in $\mathrm{I} / 32$ cases $(\mathrm{p}<0.05)$ manipulated in proestrus. This phenomenon is related to autoselection resulting from initial effect on the length of the cycle in females treated in proestrus.

\section{RÉFÉRENCES BIBLIOGRAPHIQUES}

Ajika K, Kalra S. P., Fawcett C. P., Krulich L., McCany S. M., 1972. The effects of stress and nembutal on plasma levels of gonadotropins and prolactin in ovariectomized rats. Endocrinology, 90, 707-715.

Aron C., Marx C., Marescaux J., I948. Caractère réflexe neuro-endocrinien, de l'hyperactivité réactionnelle de l'ovaire restant après hémicastration chez le Cobaye. C. R. Soc. Biol., 142, I558-1560.

Buffler G., I973. Étude du rôle joué par le cortex surrénal dans l'allongement du cycle œstral provoqué par LH chez la Ratte. C. R. Soc. Biol., 167, 1455-I458.

Feder H. H., Brown-Grant K., Corker C. S., I97i. Preovulatory progesterone, the adrenal cortex and the "critical period " for luteinizing hormone release in rats. J. Endocr., 50, 29-39.

LAwTON I., 1972. Facilitatory feedback effects of adrenal and ovarian hormones on LH secretion. Endocrinology, 90, 575-579.

Roos M., Roos J., rg66 a. Déclenchement de la ponte par l'hémicastration chez des rattes présentant des cycles de 5 jours. C. R. Acad. Sci., 262, 300-302.

Roos M., Roos J., I966 b. Mise en évidence d'un déterminisme neuro-endocrinien de la ponte provoquée par l'hémicastration chez la Ratte. C. R. Soc. Biol., 160, 647-65o. 